\title{
SCALAR CURVATURE, INEQUALITY AND SUBMANIFOLD
}

\author{
BANG-YEN CHEN AND MASAFUMI OKUMURA
}

\begin{abstract}
Using an inequality relation between scalar curvature and length of second fundamental form, we may conclude that a submanifold must have nonnegative (or positive) sectional curvatures. An application to compact submanifolds in obtained.
\end{abstract}

1. Statement of results. ${ }^{1}$ Let $M$ be an $n$-dimensional submanifold of an $(n+p)$-dimensional Riemannian manifold $N$ of constant sectional curvature $c$, and let $h$ and $H$ be the second fundamental form and the mean curvature vector field respectively. Let $h_{i j}^{\alpha}, i, j=1, \cdots, n, \alpha=$ $n+1, \cdots, n+p$, be the coefficients of the second fundamental form $h$ with respect to a local field of orthonormal frame $e_{1}, \cdots, e_{n}, e_{n+1}, \cdots$, $e_{n+p}$. Then the square of length of second fundamental form, $S$, and the scalar curvature, $R$, of $M$ are given respectively by

$$
\begin{aligned}
& S=\sum_{\alpha=n+1}^{n+p} \sum_{i, j=1}^{n}\left(h_{i j}^{\alpha}\right)^{2}, \\
& R=n^{2} H \cdot H-S+n(n-1) c,
\end{aligned}
$$

where dot "." denotes the scalar product of vectors. $A$ normal vector field $\eta$ is said to be parallel if $D \eta=0$ identically, where $D$ denotes the connection of the normal bundle. The purpose of this paper is to show the following

THEOREM 1. Let $M$ be an n-dimensional submanifold of a Riemannian manifold $N$ of constant curvature $c$. If the scalar curvature $R$ satisfies

$$
\begin{aligned}
& R \geqq(n-2) S+(n-2)(n-1) c \\
& \quad(\text { resp. } R>(n-2) S-(n-2)(n-1) c)
\end{aligned}
$$

at a point $p \in M$, then the sectional curvatures of $M$ are nonnegative (resp. positive) at $p$.

Received by the editors May 2, 1972 and, in revised form, July 31, 1972.

AMS (MOS) subject classifications (1970). Primary 53B25, 53C40.

Key words and phrases. Scalar curvature, length of second fundamental form, sectional curvature, parallel normal vector field, minimal submanifold.

${ }^{1}$ For notations and formulas we refer to [2].

(c) American Mathematical Society 1973 
THEOREM 2. Let $M$ be an n-dimensional compact submanifold of euclidean $(n+p)$-space $E^{n+p}$. Then the mean curvature vector $H$ is parallel and we have $R>(n-2) S$ if and only if $M$ is a hypersphere of a linear $(n+1)$ subspace of $E^{n+p}$ when $n \geqq 3$, and $M$ is a minimal surface of a hypersphere of $E^{n+p}$ with positive Gaussian curvature when $n=2$.

REMARK 1. If the connection of the normal bundle is flat, $n>2$, or if the submanifold is a hypersurface, Theorem 2 was proved by one of the present authors ([3], [4]).

2. Proof of Theorem 1. First we state the following lemma which is a slight generalization of a lemma given in [4]. The method of the proofs are quite the same.

Lemma. Let $a_{1}, \cdots, a_{n}, b$ be $n+1(n \geqq 2)$ real numbers satisfying the following inequality:

$$
\left(\sum_{i=1}^{n} a_{i}\right)^{2} \geqq(n-1) \sum_{i=1}^{n} a_{i}^{2}+b \quad(\text { resp. }>) ;
$$

then, for any distinct $i, j ; 1 \leqq i<j \leqq n$, we have

$$
2 a_{i} a_{j} \geqq b /(n-1) \quad(\text { resp. }>) \text {. }
$$

This lemma is proved in the following way: (4) can be rewritten as

$$
(n-2) a_{n}^{2}-2\left(\sum_{i=1}^{n-1} a_{i}\right) a_{n}+\left[(n-2) \sum_{i=1}^{n-1} a_{i}^{2}-2 \sum_{i<j<n} a_{i} a_{j}+b\right] \leqq 0,
$$

(resp. <). Denote the left-hand side by $-r$. Since $a_{n}$ is real,

Hence we obtain

$$
\begin{aligned}
\left(\sum_{i=1}^{n-1} a_{i}\right)^{2} & \left.\geqq(n-2)\left[(n-2) \sum_{i=1}^{n-1} a_{i}^{2}-2 \sum_{i<j<n} a_{i} a_{j}+b+r\right)\right] \\
& \geqq(n-2)\left[(n-1) \sum_{i=1}^{n-1} a_{i}^{2}-\left(\sum_{i=1}^{n-1} a_{i}\right)^{2}+b\right] .
\end{aligned}
$$

$$
\left.\left(\sum_{i=1}^{n-1} a_{i}\right)^{2} \geqq(n-2) \sum_{i=1}^{n-1} a_{i}^{2}+\left(\frac{n-2}{n-1}\right) b \quad \text { (resp. }>\right) .
$$

Continuing the same process $(n-2)$ times, we obtain (5).

Substituting (2) into (3), we obtain

$$
n^{2} H \cdot H \geqq(n-1) S-2(n-1) c \quad(\text { resp. }>\text { ) at } p .
$$

For simplicity we may choose a local field of orthonormal frame $e_{1}, \cdots$, $e_{n}, e_{n+1}, \cdots, e_{n+p}$ around $p$ such that $e_{n+1}$ is parallel to the mean curvature vector $H$ and $e_{1}, \cdots, e_{n}$ are in the principal directions of $e_{n+1}$ at 
$p \in M$. (If $H=0$ at $p$, we may choose an arbitrary $e_{n+1}$.) Then we have

$$
\left(h_{i j}^{n+1}\right)=\left(\begin{array}{cccc}
h_{1} & & & \\
& \cdot & & 0 \\
0 & & \cdot & \\
& & & h_{n}
\end{array}\right), \quad n^{2} H \cdot H=\left(\sum_{i=1}^{n} h_{i}\right)^{2} \text { at } p .
$$

Thus we obtain from (6):

$$
\left(\sum_{i=1}^{n} h_{i}\right)^{2} \geqq(n-1) \sum_{i=1}^{n} h_{i}^{2}+(n-1) \sum_{\alpha=n+2}^{n+p} \sum_{i, j=1}^{n}\left(h_{i j}^{\alpha}\right)^{2}-2(n-1) c
$$

Applying the lemma to (8), we get

$$
\begin{aligned}
2 h_{i} h_{j} & \geqq \sum_{\alpha=n+2}^{n+p} \sum_{k, m=1}^{n}\left(h_{k m}^{\alpha}\right)^{2}-2 c \\
& \geqq \sum_{\alpha=n+2}^{n+p}\left[\left(h_{i i}^{\alpha}\right)^{2}+\left(h_{j j}^{\alpha}\right)^{2}+2\left(h_{i j}^{\alpha}\right)^{2}\right]-2 c \\
& \geqq 2 \sum_{\alpha=n+2}^{n+p}\left[h_{i i}^{\alpha} h_{j j}^{\alpha}+\left(h_{i j}^{\alpha}\right)^{2}\right]-2 c,
\end{aligned}
$$

for any $1 \leqq i<j \leqq n$ at $p$. Thus the sectional curvature at $p$,

$$
K_{i j}=\sum_{\alpha=n+1}^{n+p}\left[h_{i i}^{\alpha} h_{j j}^{\alpha}-\left(h_{i j}^{\alpha}\right)^{2}\right]+c,
$$

for the plane section spanned by $e_{i}$ and $e_{j}$ is nonnegative (resp. positive). This proves the theorem.

3. Proof of Theorem 2. Let $M$ be an $n$-dimensional submanifold of an $(n+p)$-dimensional Riemannian manifold $N$ of constant sectional curvature $c$ and $\eta$ be a parallel unit normal vector over $M$. If we choose the local fields of orthonormal frame in such a way that $e_{n+1}=\eta$ and $e_{1}, \cdots, e_{: 2}$ are in the principal directions of $e_{n+1}$, then we have

$$
H_{n+1}=\left(\begin{array}{cccc}
h_{1} & & & 0 \\
& \cdot & & \\
& & \cdot & \\
0 & & & h_{n}
\end{array}\right) \text {. }
$$

We assume that $\operatorname{Tr} H_{n+1}$ is constant. Then a recent paper of Smyth [5] gives the following formula:

$$
\sum_{i, j=1}^{n} h_{i j}^{n+1} \Delta h_{i j}^{n+1}=\sum_{i<j}\left[K_{i j}+\sum_{\beta}\left(h_{i j}^{\beta}\right)^{2}\right]\left(h_{i}-h_{j}\right)^{2},
$$


where $\Delta h_{i j}^{n+1}$ denotes the Laplacian of the second fundamental form $h_{i j}^{n+1}$ in the direction of $e_{n+1}$. Now, suppose that $M$ is an $n$-dimensional compact submanifold of $E^{n+p}$ such that the mean curvature vector $H$ is parallel and $R>(n-2) S$. Then, by Theorem 1 , we see that the sectional curvatures of $M$ are all positive, that is, $K_{i j}>0$ for $1 \leqq i<j \leqq n$. Therefore, we see that $\sum_{i, j} h_{i j}^{n+1} \Delta h_{i j}^{n+1} \geqq 0$. Hence we get

$$
\frac{1}{2} \Delta\left(\operatorname{Tr} H_{n+1}^{2}\right)=\sum_{i, j} h_{i j}^{n+1} \Delta h_{i j}^{n+1}+\sum_{i, j, k}\left(h_{i j k}^{n+1}\right)^{2} \geqq 0 .
$$

By Hopf's lemma we see that $h_{i j k}^{n+1}=0$ and $\sum_{i, j} h_{i j}^{n+1} \Delta h_{i j}^{n+1}=0$. Hence, from (10) we have

$$
h_{1}=\cdots=h_{n} \neq 0 \text {. }
$$

This shows that $M$ is pseudo-umbilical in $E^{n+p}$ and $H$ is parallel. Hence, we see that $M$ is contained in a hypersphere $S^{n+p-1}$ of $E^{n+p}$ as a minimal submanifold (see, for instance, [1]). Without loss of generality, we may assume that $S^{n+p-1}$ is of radius 1 . Then, by the assumption, $R>(n-2) S$, we see that the square of the length of second fundamental form of $M$ in $S^{n+p-1}$, say $\bar{S}$, satisfies

$$
S<n /(n-1) \text {. }
$$

Therefore, by a result of Chern-do Carmo-Kobayashi [2], we find that if $n \geqq 3$, then $M$ must be totally geodesic in $S^{n+p-1}$. Hence $M$ is a hypersphere of a linear $(n+1)$-subspace of $E^{n+p}$. If $n=2$, then the condition $R>(n-2) S$ implies that the Gaussian curvature of $M$ is positive. This proves a part of the theorem. The remaining part is obvious.

\section{REFERENCES}

1. B.-Y. Chen, On the mean curvature of submanifolds of euclidean space, Bull. Amer. Math. Soc. 77 (1971), 741-743. MR 44 \#2177.

2. S. S. Chern, M. do Carmo and S. Kobayashi, Minimal submanifolds of a sphere with second fundamental form of constant length, Functional Analysis and Related Fields (Proc. Conf. for M. Stone, Univ. of Chicago, Chicago, Ill., 1968), Springer, New York, 1970, pp. 59-75. MR 42 \#8424.

3. M. Okumura, Hypersurfaces and a pinching problem on the second fundamental tensor (to appear).

4. - Submanifolds and a pinching problem on the second fundamental tensor (to appear).

5. B. Smyth, Submanifolds of constant mean curvature (to appear).

Department of Mathematics, Michigan State University, East Lansing, MICHIGAN 48823 (Current address of Bang-yen Chen)

Current address (Masafumi Okumura): Department of Mathematics, Saitama University, Tokyo, Japan 